ARTICLE

Received 10 Mar 2016 | Accepted 17 Jun 2016 | Published 22 Jul $2016 \quad$ DOl: 10.1038/ncomms12272 OPEN

\title{
A rhodium/silicon co-electrocatalyst design concept to surpass platinum hydrogen evolution activity at high overpotentials
}

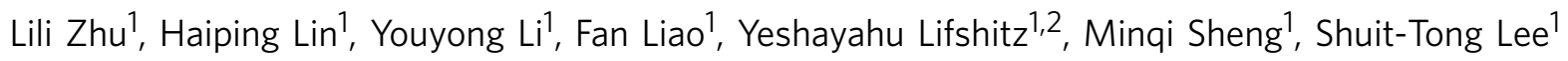
\& Mingwang Shao ${ }^{1}$

Currently, platinum-based electrocatalysts show the best performance for hydrogen evolution. All hydrogen evolution reaction catalysts should however obey Sabatier's principle, that is, the adsorption energy of hydrogen to the catalyst surface should be neither too high nor too low to balance between hydrogen adsorption and desorption. To overcome the limitation of this principle, here we choose a composite (rhodium/silicon nanowire) catalyst, in which hydrogen adsorption occurs on rhodium with a large adsorption energy while hydrogen evolution occurs on silicon with a small adsorption energy. We show that the composite is stable with better hydrogen evolution activity than rhodium nanoparticles and even exceeding those of commercial platinum/carbon at high overpotentials. The results reveal that silicon plays a key role in the electrocatalysis. This work may thus open the door for the design and fabrication of electrocatalysts for high-efficiency electric energy to hydrogen energy conversion.

\footnotetext{
${ }^{1}$ Institute of Functional Nano and Soft Materials (FUNSOM), Jiangsu Key Laboratory for Carbon-Based Functional Materials and Devices, Soochow University, Suzhou, Jiangsu, 215123, China. ${ }^{2}$ Materials Science and Engineering Department, Technion, Israel Institute of Technology, Haifa 3200003 , Israel. Correspondence and requests for materials should be addressed to Y.L. (email: yyli@suda.edu.cn) or to S.-T.L. (email: apannale@suda.edu.cn) or to M.S. (email: mwshao@suda.edu.cn).
} 
$\mathrm{H}$ ydrogen is considered to be one of the most important future sources of clean energy. Efficient and environmentally friendly methods for hydrogen production are thus extensively investigated. Electrolysis of water is one of the simplest and most advanced ways of hydrogen evolution ${ }^{1,2}$ which is currently being commercially used. This is why extensive research is still focused on the development of electrocatalysts with improved hydrogen evolution reaction (HER) efficiency.

A host of candidate materials for HER electrocatalytic applications were studied including metals ${ }^{1}$, sulfides ${ }^{2-5}$ and different composites (metals/sulfides ${ }^{6}$, sulfides/selenides ${ }^{7}, \mathrm{C}_{3} \mathrm{~N}_{4} /$ graphene $^{8}$ and $\mathrm{Co} /$ graphene ${ }^{9}$ ). Platinum $(\mathrm{Pt})$ is considered 'the Holy Grail' of HER electrocatalysts, since Pt-based electrocatalysts have the best performance for hydrogen evolution, supplying fast kinetic rate and near-zero overpotential ${ }^{7,10}$.

One of the oldest rules in catalysis, Sabatier's principle ${ }^{11}$ states that for efficient HER the adsorption energy should be neither too high nor too low, because if it is too high (endothermic) adsorption is slow and the overall rate is slow as well. If it is too low (exothermic) desorption is slow ${ }^{2,12}$. To overcome the limitation of Sabatier's principle, we decided to separate the adsorbing surface and the desorbing surface by applying a metal/ SiNW composite. Strong adsorption of hydrogen on the metal surface allows a large overall reaction rate, while high desorption from the SiNW surface allows a large evolution rate. We study several pure metals or metal/C composites as well as several metal/SiNW composites. The HER electrocatalytic activity of the metal/SiNW is found better than the pure metal electrode activity, supporting the validity of the composite concept for all metals investigated. More specifically, the HER activity of Rh/SiNW even surpasses that of $\mathrm{Pt} / \mathrm{C}$ at sufficiently high overpotentials. Linear scan voltammetry (LSV) and hydrogen evolution measurements show that $\mathrm{Rh} / \mathrm{SiNW}$ electrodes have a higher electricity-tohydrogen-energy conversion efficiency than the commercial $\mathrm{Pt} / \mathrm{C}$ electrodes. Moreover, at a high current density of $1,000 \mathrm{~mA} \mathrm{~cm}^{-2}$ the stability of hydrogen generation of the $\mathrm{Rh} / \mathrm{SiNW}$ electrode is better than that of a $\mathrm{Pt} / \mathrm{C}$ electrode. To support our experimental findings we also perform density functional theory (DFT) simulations of the Rh/SiNW system, which confirm the benefits of our composite approach of dividing the catalyst into two separate surfaces with large and small adsorption energies, respectively. The DFT calculations also show that $\mathrm{Si}$ poisoning by a hydroxyl can be removed by Rh-adsorbed hydrogen, which stabilizes the $\mathrm{Rh} / \mathrm{SiNW}$ catalyst performance. This work thus opens the door for the design and fabrication of electrocatalysts with improved electric energy to hydrogen energy conversion efficiency.

\section{Results}

Structural characterization. LSV measurements were performed to probe the HER activity of the studied electrodes applying a typical three electrodes electrochemical workstation in an acidic medium (oxygen free $0.5 \mathrm{M} \mathrm{H}_{2} \mathrm{SO}_{4}$ serving as the electrolyte). The electrodes studied included commercial $\mathrm{Pt} / \mathrm{C}$ electrodes (20 and $40 \mathrm{wt} \% \mathrm{Pt}$ ) representing the best currently available HER electrocatalyst. Several metal/SiNW electrodes were prepared with different concentrations of metals (denoted as $x \mathrm{wt} \% \mathrm{M} / \mathrm{SiNW}$ with $x$ being the relative mass of $\mathrm{M}$ in the composite).

SiNWs were prepared employing the oxide-assisted growth method ${ }^{13}$. Rh/SiNW was obtained by reducing $\mathrm{Rh}^{3+}$ ion via in situ growth of Rh nanoparticles (NPs) on the surface of hydrogen-terminated $\mathrm{SiNWs}^{14}$. All other M/SiNWs were obtained in a similar way. Supplementary Figs 1 and 2 are the $\mathrm{X}$-ray diffraction pattern and X-ray photoelectron spectroscopy spectra of $29.1 \mathrm{wt} \% \mathrm{Rh} / \mathrm{SiNWs}$, revealing their crystal structure and composition. Transmission electron microscopy (TEM) image (Fig. 1a) shows that the $29.1 \mathrm{wt} \% \mathrm{Rh} / \mathrm{SiNW}$ consists of a SiNW of $40 \mathrm{~nm}$ in diameter wrapped by Rh NPs. High-resolution TEM enlargement (Fig. 1b) of the red square in Fig. 1a shows a $\mathrm{Rh}$ NP embedded in the SiNW with the lattice spacing of $0.22 \mathrm{~nm}$ corresponding to the (111) interplanar distance of cubic Rh. High-angle annular dark field scanning TEM image of a single $\mathrm{Rh}$ NP attached to SiNW (Fig. 1c) and energy dispersive spectroscopy mapping show the elemental distribution of oxygen (red), silicon (orange) and rhodium (green), respectively (Fig. 1d).

Electrochemical characterization. Figure 2a shows LSV curves of electrodes made of different metals/SiNW with a metal weight ratio of $\sim 30 \%$. The best HER activities are obtained for $\mathrm{Rh} /$ SiNW and Pt/SiNW. Pt/SiNW has a slightly lower overpotential for a current density of $30 \mathrm{~mA} \mathrm{~cm}^{-2}$. The current increase with potential is however larger for Rh/SiNW than for $\mathrm{Pt} / \mathrm{SiNW}$ so that the overpotential for $100 \mathrm{~mA} \mathrm{~cm}^{-2}$ is $0.18 \mathrm{~V}$ for $\mathrm{Rh} / \mathrm{SiNW}$ compared with $0.22 \mathrm{~V}$ for Pt/SiNW. The LSV curves show the corresponding pure metals (Supplementary Fig. 3) to have a smaller HER activity than the M/SiNW composites validating that the composite concept of separating the adsorbing and desorbing site is indeed correct. Figure $2 \mathrm{~b}$ presents the effect of the Rh concentration on the HER activity of Rh/SiNW catalysts, showing clearly that the HER activity for lower (0.4 and 9) wt\% of $\mathrm{Rh}$ is worse than that of pure Rh NPs, whereas the HER activity for larger (19.6 and 29.1) wt\% surpasses that of the pure Rh. The HER catalytic activity increases with increase in Rh content from 0.4 to $59.9 \mathrm{wt} \%$ and a further increment in Rh content (85.5 wt\%) leads to decrease in the catalytic activity. This is due to the

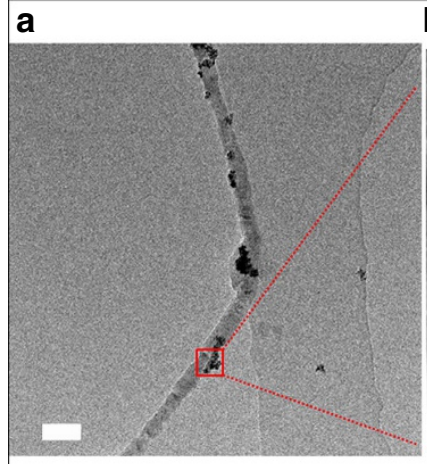

C

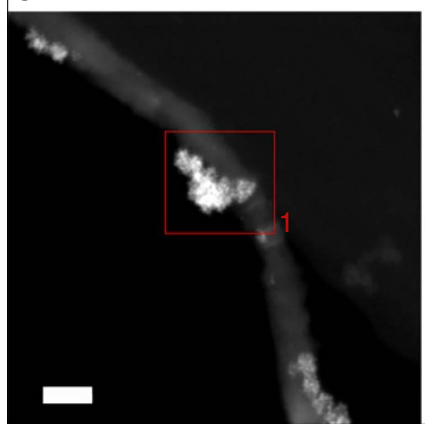

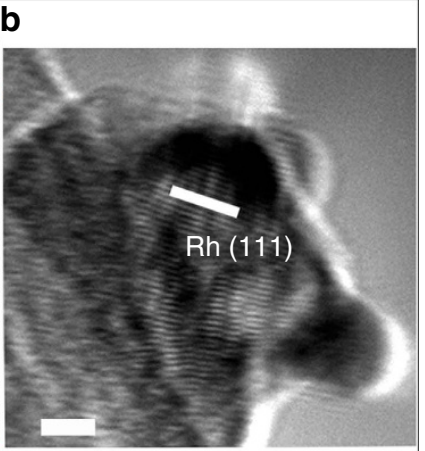
d

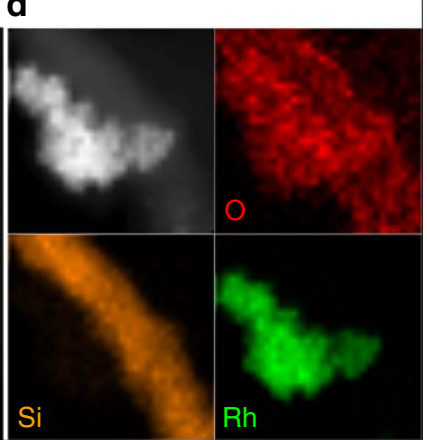

Figure 1 | TEM characterization. (a) TEM image of a Rh/SiNW. Scale bar, $100 \mathrm{~nm}$. (b) Enlarged HRTEM image of the red square in a showing a Rh crystallite. The $0.22 \mathrm{~nm}$ spacing corresponds to Rh (111). Scale bar, $5 \mathrm{~nm}$. (c) HAADF-STEM image of a Rh/SiNW. Scale bar, $50 \mathrm{~nm}$. (d) Its corresponding EDS mapping showing the $\mathrm{O}$, Si and Rh distributions. EDS, energy dispersive spectroscopy; HAADF-STEM, high-angle annular dark field scanning TEM; HRTEM, high-resolution TEM. 
a

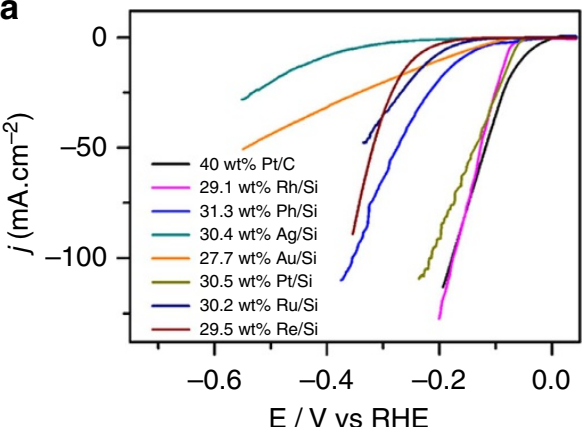

C

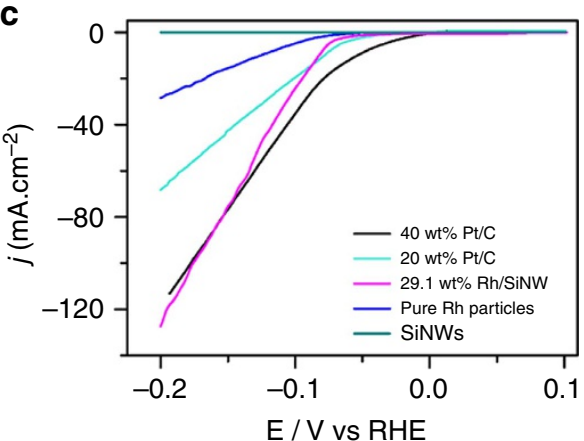

b

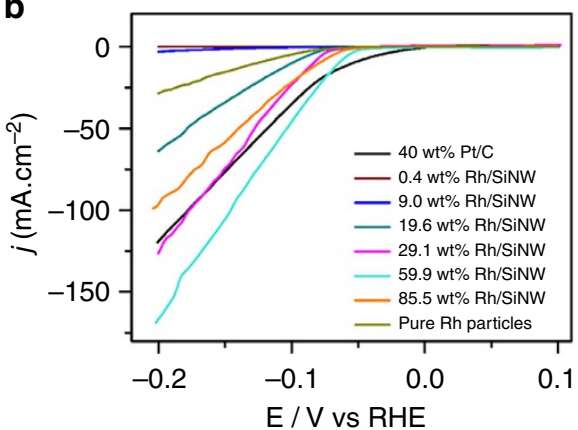

d

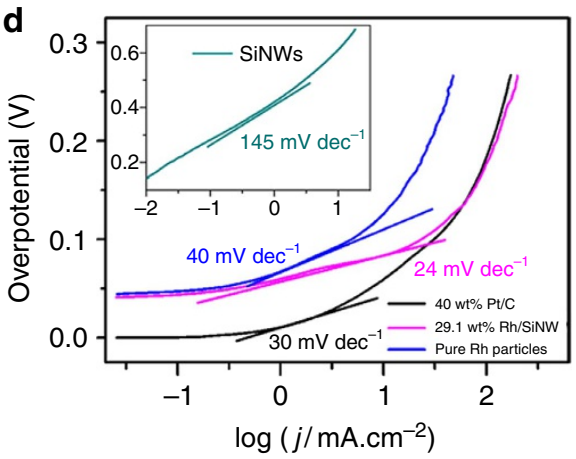

Figure 2 | The electrochemical activity of different electrocatalysts in oxygen-free $\mathbf{0 . 5} \mathbf{~ M ~ \mathbf { H } _ { 2 } \mathbf { S O }} \mathbf{4}_{\mathbf{4}}$. (a) LSV plots of metal/SiNW catalysts containing about $30 \mathrm{wt} \%$ metal. The metal type is indicated. The LSV of $40 \mathrm{wt} \% \mathrm{Pt} / \mathrm{C}$ is also shown. $29.1 \mathrm{wt} \% \mathrm{Rh} / \mathrm{SiNW}$ has the best HER activity of all metal/SiNW catalysts. (b) LSV plots of Rh/SiNW catalysts with different Rh wt\% with the 59.9 wt\% the best one. (c) LSV plots of SiNWs, pure Rh, 29.1 wt\% Rh/SiNW, and 20 and $40 \mathrm{wt} \% \mathrm{Pt} / \mathrm{C}$. The HER activity of $40 \mathrm{wt} \% \mathrm{Pt} / \mathrm{C}$ is better than that of $20 \mathrm{wt} \% \mathrm{Pt} / \mathrm{C}$, so only the $40 \mathrm{wt} \% \mathrm{Pt} / \mathrm{C}$ data was used as a reference to the Rh/SiNW data. The HER activity of $29.1 \mathrm{wt} \% \mathrm{Rh} / \mathrm{SiNW}$ is worse than that of $40 \mathrm{wt} \% \mathrm{Pt} / \mathrm{C}$ for $\mathrm{V}>-160 \mathrm{mV}$ but is better for $\mathrm{V}<-160 \mathrm{mV}$. (d) Tafel plots and Tafel slopes derived from c. The Tafel slopes are 145, 40, 30 and $24 \mathrm{mV} \mathrm{dec}^{-1}$ for SiNWs, pure Rh, $40 \mathrm{wt} \% \mathrm{Pt} / \mathrm{C}$ and $29.1 \mathrm{wt} \% \mathrm{Rh} / \mathrm{SiNW}$, respectively. The Tafel slope of $29.1 \mathrm{wt} \% \mathrm{Rh} / \mathrm{SiNW}$ is smaller than that of $40 \mathrm{wt} \% \mathrm{Pt} / \mathrm{C}$ indicating the better HER performance of $29.1 \mathrm{wt} \% \mathrm{Rh} / \mathrm{SiNW}$.

synergistic effect of adsorption on the Rh surface and desorption from the Si surface.

Figure 2c compares the LSV curves of pure SiNWs, pure Rh NPs, 20 and $40 \mathrm{wt} \% \mathrm{Pt} / \mathrm{C}$ (commercial catalysts) and $29.1 \mathrm{wt} \%$ $\mathrm{Rh} / \mathrm{SiNW}$. The 20 and $40 \mathrm{wt} \% \mathrm{Pt} / \mathrm{C}$ catalysts show nearly theoretical value $(0 \mathrm{~V}$ versus $\mathrm{RHE})$ for onset potential of hydrogen evolution. The $40 \mathrm{wt} \% \mathrm{Pt} / \mathrm{C}$ catalyst shows better HER characteristics than the $20 \mathrm{wt} \% \mathrm{Pt} / \mathrm{C}$, so only the $40 \mathrm{wt} \%$ catalyst was subsequently used as a reference to the other catalysts. The $29.1 \mathrm{wt} \% \mathrm{Rh} / \mathrm{SiNW}$ and pure Rh NPs catalysts need 44 and $47 \mathrm{mV}$ to sustain a current density of $0.1 \mathrm{~mA} \mathrm{~cm}^{-2}$. The SiNWs show negligible HER activity. It is interesting to note that the cathodic current of the $29.1 \mathrm{wt} \% \mathrm{Rh} / \mathrm{SiNW}$-modified glassy carbon (GC) electrode increases with increasing overpotential much faster than that of the $40 \mathrm{wt} \% \mathrm{Pt} / \mathrm{C}$. The exchange current densities of $29.1 \mathrm{wt} \% \mathrm{Rh} / \mathrm{SiNW}$, pure $\mathrm{Rh}$ and $40 \mathrm{wt} \% \mathrm{Pt} / \mathrm{C}$ were calculated to be $0.00858,0.0222$ and $0.388 \mathrm{~mA} \mathrm{~cm}^{-2}$ (Supplementary Table 1). The $40 \mathrm{wt} \% \mathrm{Pt} / \mathrm{C}$ is the best one; the value ratio and $\mathrm{Rh}$ mass ratio of $29.1 \mathrm{wt} \% \mathrm{Rh} / \mathrm{SiNW}$ to pure $\mathrm{Rh}$ are 0.396 and 0.291 , respectively, which indicates the exchange current density of $29.1 \mathrm{wt} \% \mathrm{Rh} / \mathrm{SiNW}$ is mainly from $\mathrm{Rh}$ active part.

The cathodic current of $29.1 \mathrm{wt} \% \mathrm{Rh} / \mathrm{SiNW}$ catalysts thus exceeds that of the $40 \mathrm{wt} \% \mathrm{Pt} / \mathrm{C}$ ones when the potential is more negative than $-160 \mathrm{mV}$ (the current is $80 \mathrm{mAcm}^{-2}$ at the intersection of these two curves). Neither SiNWs nor pure Rh catalysts show a better electrocatalytic performance than $\mathrm{Pt} / \mathrm{C}$. It is thus reasonable to deduce that the improved HER performance of the composite material is due to a synergistic electrocatalytic effect in the $\mathrm{Rh} / \mathrm{SiNW}$ system. The $\mathrm{Rh} / \mathrm{SiNW}$ catalysts are more advantageous than the conventional $\mathrm{Pt} / \mathrm{C}$ electrodes for practical applications, since the production of hydrogen in industry requires current densities in the order of $1,000 \mathrm{~mA} \mathrm{~cm}^{-2}$ (ref. 15). The overpotentials of the $29.1 \mathrm{wt} \%$ $\mathrm{Rh} / \mathrm{SiNW}$ nanocomposite and $40 \mathrm{wt} \% \mathrm{Pt} / \mathrm{C}$ catalyst are 0.95 and $1.12 \mathrm{~V}$, respectively, at $1,000 \mathrm{~mA} \mathrm{~cm}^{-2}$ (Fig. 3a) with hydrogen to electric energy efficiencies of $56.4 \%$ and $52.3 \%$ (for details see Supplementary Note 1), respectively. Note that at high current densities, the LSV curve for the $29.1 \mathrm{wt} \% \mathrm{Rh} / \mathrm{SiNW}$ electrode is smooth and stable, whereas it is oscillatory and less stable for the $40 \mathrm{wt} \% \mathrm{Pt} / \mathrm{C}$ electrode.

To further substantiate the improved efficiency for hydrogen generation obtained by the Rh/SiNW system, we compared the hydrogen evolution of the $40 \mathrm{wt} \% \mathrm{Pt} / \mathrm{C}$ and the $29.1 \mathrm{wt} \% \mathrm{Rh} /$ SiNW systems at the same potential of $-0.4 \mathrm{~V}$. Figure $3 \mathrm{~b}$ shows that the hydrogen evolution of the Rh/SiNW system was larger by $15 \%$.

The synergistic HER reaction. The curves in Fig. 2c were re-plotted to get the Tafel plots and the Tafel slopes (Fig. 2d) to quantitatively compare the HER electrocatalytic activities.

The Tafel equation:

$$
V=\mathrm{a}+\mathrm{b} \cdot \log (-j)
$$

(where $V$ is the overpotential, $j$ the current density and $\mathrm{b}$ is the Tafel slope) is derived from the electrochemical kinetics, and reveals the relation between the electrochemical reaction rate and the overpotential. The Tafel slope is an important indicator in electrochemistry for the increase of the electrochemical current (that is, the reaction rate) upon an incremental increase of the overpotential. Small slopes represent a better electrocatalyst, since 

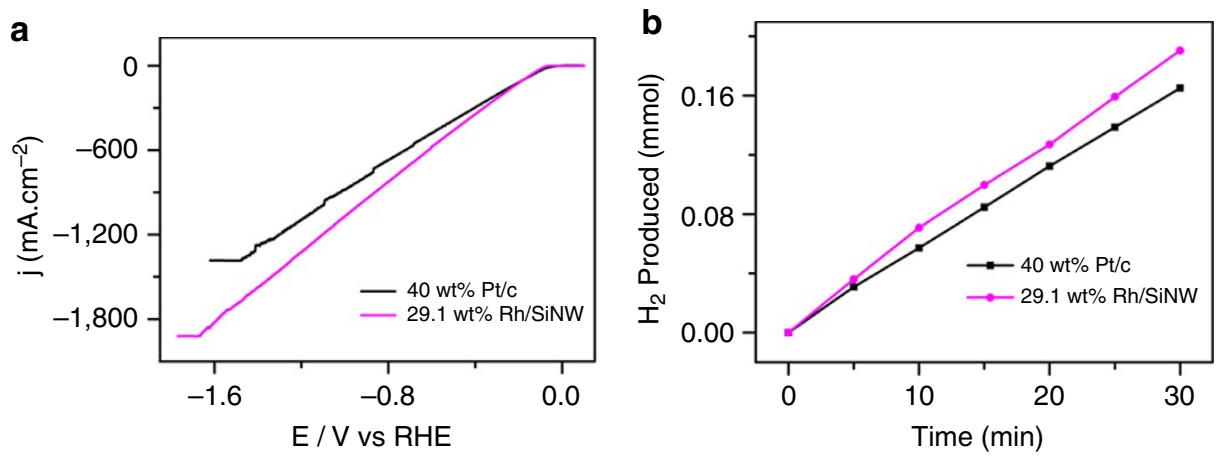

Figure 3 | HER relative efficiency of $\mathbf{2 9 . 1}$ wt\% Rh/SiNW and $\mathbf{4 0}$ wt\% Pt/C in oxygen-free $\mathbf{0 . 5} \mathbf{M ~ H}_{\mathbf{2}} \mathbf{S O}_{\mathbf{4}}$. (a) LSV of both catalysts for high current densities. At $1,000 \mathrm{~mA} \mathrm{~cm}^{-2}$ the electric power to hydrogen energy conversion efficiency of $29.1 \mathrm{wt} \% \mathrm{Rh} / \mathrm{SiNW}$ is larger by $7.8 \%$. Note that the current for $29.1 \mathrm{wt} \% \mathrm{Rh} / \mathrm{SiNW}$ is more stable. (b) Hydrogen evolution by $29.1 \mathrm{wt} \% \mathrm{Rh} / \mathrm{SiNW}$ and $40 \mathrm{wt} \% \mathrm{Pt} / \mathrm{C}$ systems at $-0.4 \mathrm{~V}$. The hydrogen evolution using $29.1 \mathrm{wt} \% \mathrm{Rh} / \mathrm{SiNW}$ is larger by $15 \%$.

a

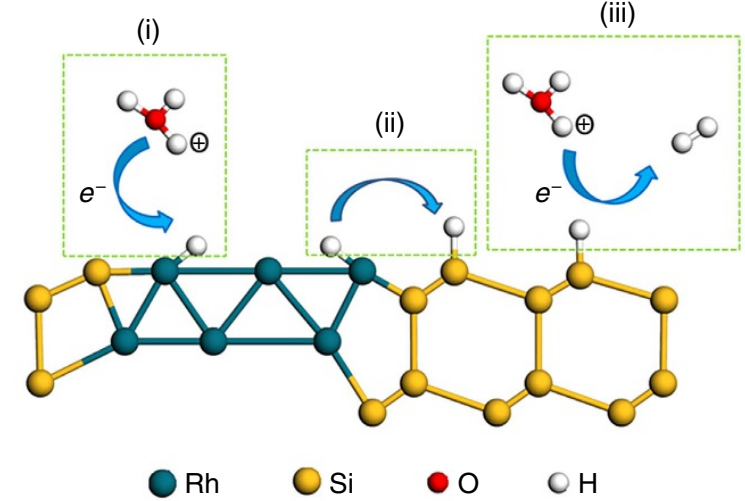

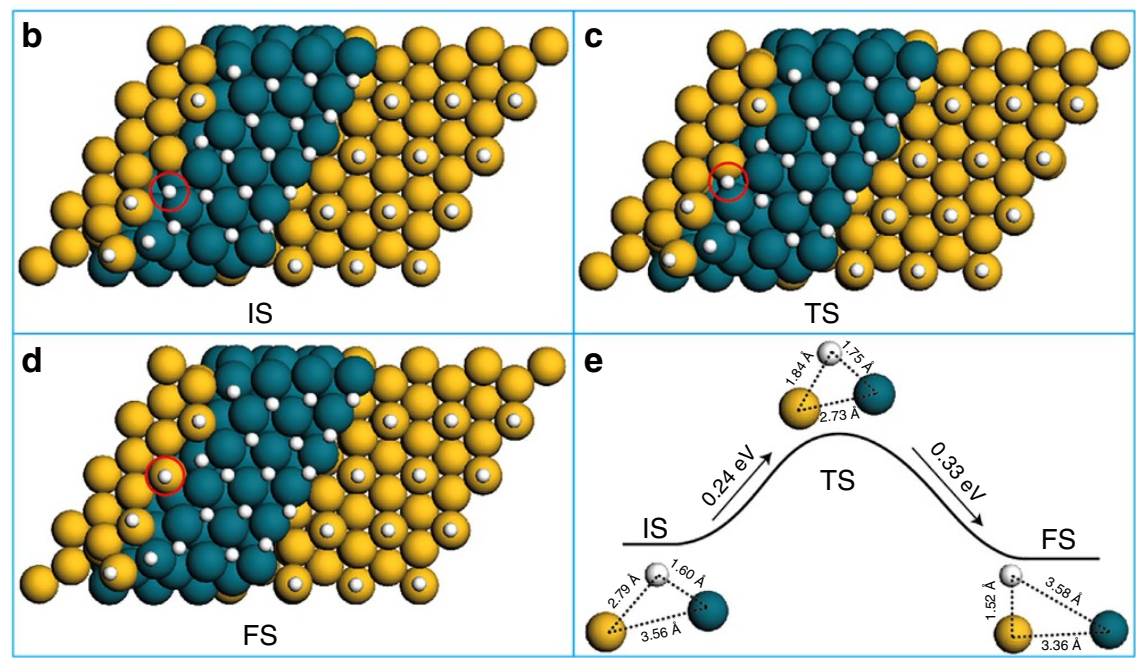

Figure 4 | Modelling of the HER reaction on Rh/SiNW. (a) A schematic representation of the HER reaction mechanism: (i) adsorption of hydrogen ions on the Rh surface. (ii) Diffusion of a hydrogen atom from the Rh surface to the Si surface. (iii) The adsorbed hydrogen atom on the Si surface reacts with a proton to form a hydrogen molecule. And the Rh-adsorbed hydrogen may migrate to the surface of $\mathrm{Si}$ atoms (marked with a red circle): (b) IS, initial state; (c) TS, transition state; and (d) FS, final state. (e) The activation energy to get to the transition state is $0.24 \mathrm{eV}$ and in the final state the adsorbed hydrogen is $0.33 \mathrm{eV}$ below the transition state and $0.09 \mathrm{eV}$ below the initial state.

for small slopes the current gain with potential increase would be larger.

The Tafel slopes (Fig. 2d) for SiNW-, pure Rh- and Pt/Cmodified GC electrodes derived from Fig. 2c are 145 (inset), 40 and $30 \mathrm{mV} \mathrm{dec}^{-1}$, respectively, whereas the Tafel slope of $\mathrm{Rh} /$ SiNW catalysts is as low as $24 \mathrm{mV} \mathrm{dec}^{-1}$. The Tafel slopes (Fig. 2d) show the $29.1 \mathrm{wt} \% \mathrm{Rh} / \mathrm{SiNW}$ to have the best HER kinetics among all electrocatalysts studied, since a smaller Tafel slope signifies a more rapid increase of the HER rate with increasing overpotential. The above discussion shows that $\mathrm{Rh} /$ SiNW not only has a catalytic activity comparable to $\mathrm{Pt} / \mathrm{C}$ at low overpotentials $(0$ to $-160 \mathrm{mV})$, but also is an excellent catalyst, even better than $\mathrm{Pt} / \mathrm{C}$ in practical working potentials (more negative than $-160 \mathrm{mV}$ versus RHE). 
There are three widely-accepted HER mechanisms. ${ }^{16}$ The Volmer mechanism:

$$
\mathrm{H}^{+}+\mathrm{M}+e^{-} \rightarrow \mathrm{M}-\mathrm{H}
$$

The Heyrovsky mechanism:

$$
\mathrm{H}^{+}+\mathrm{M}-\mathrm{H}+e^{-} \rightarrow \mathrm{H}_{2}+\mathrm{M}
$$

The Tafel mechanism:

$$
\mathrm{M}-\mathrm{H}+\mathrm{M}-\mathrm{H} \rightarrow \mathrm{H}_{2}+2 \mathrm{M}
$$

The theoretical Tafel slopes of the three mechanisms are 120, 40 and $30 \mathrm{mV} \mathrm{dec}^{-1}$, respectively ${ }^{16}$. Comparison of the experimental Tafel slopes with the theoretical values indicate that SiNWs, pure $\mathrm{Rh}$ and $40 \mathrm{wt} \% \mathrm{Pt} / \mathrm{C}$ catalysts follow the Volmer, Heyrovsky and Tafel mechanisms, respectively, while the $\mathrm{Rh} / \mathrm{SiNW}$ catalyst (Tafel slope being $24 \mathrm{mV} \mathrm{dec}^{-1}$ ) may follow a different reaction mechanism.

We propose the following mechanism to explain the synergistic effect of the $\mathrm{Rh} / \mathrm{SiNW}$ system. We suggest that the HER process in $\mathrm{Rh} / \mathrm{SiNW}$ advances in three main steps (Fig. 4a): (i) adsorption of a hydrogen ion on the Rh surface (left part, Fig. 4a); (ii) migration of the neutral adsorbed- $\mathrm{H}$ atom to the $\mathrm{Si}$ surface (middle part, Fig. 4a); and (iii) reaction of the $\mathrm{H}$ atom with a hydrogen ion to produce a hydrogen molecule in the gas phase (right part, Fig. 4a).

The calculated adsorption free energy is also a key parameter to semi-quantitatively understand the variation in the exchange currents of catalysts ${ }^{17}$. DFT calculation was employed to obtain the difference of free energy $\left(\Delta \mathrm{G}_{\mathrm{H}^{*}}\right)$. The $\Delta \mathrm{G}_{\mathrm{H}^{*}}$ of $\mathrm{Rh} / \mathrm{Si}$ is $-0.10 \mathrm{eV}$ (Supplementary Table 4), which is very similar to the estimated on the Pt surface $(-0.09$ and $-0.03 \mathrm{eV}$ for 0.25 and 1 molecular layer, respectively ${ }^{17}$.

DFT calculations were performed to substantiate the proposed mechanism. The $\mathrm{Rh} / \mathrm{SiNW}$ catalyst was modelled with a hydrogenterminated $\mathrm{Si}(111)$ surface and a $\mathrm{Rh}(111)$ surface (Supplementary Fig. 4a). The calculations indicate that in an acidic aqueous medium, the hydrogen ions quickly adsorb on the surface of Rh (Supplementary Fig. 4b) and obtain electrons to become atomic hydrogen atoms. The Rh-adsorbed hydrogen may then migrate to bare Si surface atoms near the $\mathrm{Si} / \mathrm{Rh}$ interfaces (Figs $4 \mathrm{~b}, \mathrm{c}$ and Supplementary Movie 1). The calculated activation barrier of such a migration process is $0.24 \mathrm{eV}$ per hydrogen atom, followed by an energy drop of $0.09 \mathrm{eV}$ (Fig. 4e). The hydrogen adsorbed on the $\mathrm{Si}$ surface reacts with a hydronium ion (stabilized by water molecules in the solution) to produce a hydrogen gas molecule. This last reaction is a spontaneous process so that the rate-determining process is, therefore, the diffusion of atomic hydrogen from the $\mathrm{Rh}$ surface to the Si surface. Consequently the activation barrier of the entire HER process on $\mathrm{Rh} / \mathrm{SiNW}$ is $0.24 \mathrm{eV}$, implying an excellent catalytic activity. Note that this activation barrier is much smaller than that on pure Rh catalysts $(0.69 \mathrm{eV}$, Supplementary Fig. 5). Thus the synergistic HER process could be described by the following equations:

$$
\begin{gathered}
\mathrm{Rh}-\mathrm{Si}+\mathrm{H}^{+}+e^{-} \Leftrightarrow \mathrm{H}-\mathrm{Rh}-\mathrm{Si} \\
\mathrm{H}-\mathrm{Rh}-\mathrm{Si} \Leftrightarrow \mathrm{Rh}-\mathrm{Si}-\mathrm{H} \\
\mathrm{Rh}-\mathrm{Si}-\mathrm{H}+\mathrm{H}^{+}+e^{-} \rightarrow \mathrm{H}_{2}+\mathrm{Rh}-\mathrm{Si}
\end{gathered}
$$

The detailed calculation of these reactions kinetics is given in the Supplementary Note 2.

The Tafel slope is: $\frac{2.303 R T}{(2+\alpha) F}=0.024 \mathrm{~V} \cdot \operatorname{dec}^{-1}$ (assuming $\alpha=0.5$, $F$ is the Faraday constant, $R$ the Rydberg gas constant and $T$ the absolute temperature) in accord with the experimental data presented above.

Additional experiments provided further insight into the reaction mechanism. The pH-dependent relation of the HER was experimentally determined to be 2.13 (Supplementary Fig. 6), which is in accord with the theoretical value of 2 (Supplementary Note 2). The electrochemical impedance spectroscopy was also investigated (Supplementary Table 2 and Supplementary Fig. 7), a two-capacitive process supporting the formation of surface adsorption during the HER reaction. The electrochemical impedance spectroscopy was employed to calculate the real surface area and real current density (Supplementary Table 3) using Supplementary Methods.

The lifetime of a catalyst is an important parameter, especially when $\mathrm{Si}$ is used due to its poor stability in the oxidation environment. The $\mathrm{Rh} / \mathrm{SiNW}$ and $\mathrm{Pt} / \mathrm{C}$ catalysts were thus tested in oxygen-free $0.5 \mathrm{M} \mathrm{H}_{2} \mathrm{SO}_{4}$ at room temperature under a constant potential of $-0.1 \mathrm{~V}$ showing that the cathodic currents remained steady for $500,000 \mathrm{~s}$, indicating the long lifetime of both catalysts (Supplementary Fig. 8). The long lifetime of $\mathrm{Rh} / \mathrm{SiNW}$ catalysts is supported by their self-regeneration ability, which was found by theoretical simulations and shown in Supplementary Fig. 9. When a Si surface atom is terminated with a hydroxyl (or in other words, poisoned by a hydroxyl), a $\mathrm{Rh}$-adsorbed hydrogen may react with the hydroxyl to regenerate the $\mathrm{Si}$ atom by producing water molecules. The energy barrier of such water formation reaction is only $0.45 \mathrm{eV}$, and the energy of the final state is $0.06 \mathrm{eV}$ lower than that of the initial one. The role of $\mathrm{Rh}$ in the regeneration of $\mathrm{Si}$ active sites is crucial to maintaining excellent electrocatalysis of the composite catalysts.

\section{Discussion}

This work focuses on the improvement of the HER activity of electrocatalysts aiming to achieve better activity than that of $\mathrm{Pt} / \mathrm{C}$, which has the highest HER activity reported so far. We report an approach to overcome the limitations dictated by the Sabatier's principle (in which hydrogen adsorption and desorption occur on the same catalyst surface) by separating the adsorbing surface from the desorbing surface of HER catalysts. We design metal/ SiNW composites as the catalysts in which the metal is the strongly hydrogen adsorbing surface and $\mathrm{Si}$ is the weakly adsorbing surface offering rapid evolution of hydrogen. We demonstrate that the approach indeed works, that is, the HER activity of the metal/SiNW composite is better than that of the pure metal. $\mathrm{Rh} / \mathrm{SiNW}$ is shown to be an outstanding catalyst with HER activities exceeding those of $\mathrm{Pt} / \mathrm{C}$ for sufficiently large current densities (high overpotentials) and with a Tafel slope of $0.024 \mathrm{~V} \mathrm{dec}^{-1}$ compared with $0.03 \mathrm{~V} \mathrm{dec}^{-1}$ for Pt/C. The lower Tafel slope of Rh/SiNW explains why the LSV curves of Rh/SiNW and $\mathrm{Pt} / \mathrm{C}$ intersect, so that the HER activity of $\mathrm{Rh} / \mathrm{SiNW}$ surpasses that of $\mathrm{Pt} / \mathrm{C}$ for sufficiently high overpotentials (large current densities). Despite $\mathrm{Pt} / \mathrm{C}$ showing lower overpotentials than $\mathrm{Rh} /$ SiNW catalysts at low current densities, the electric energy to hydrogen conversion efficiency of $\mathrm{Rh} / \mathrm{SiNW}$ catalysts at high current densities of $1,000 \mathrm{~mA} \mathrm{~cm}^{-2}$ (as used by the industry) is $7.8 \%$ larger than that of $\mathrm{Pt} / \mathrm{C}$. The $\mathrm{H}_{2}$ generation rate of $\mathrm{Rh} / \mathrm{SiNW}$ at the same overpotential of $0.4 \mathrm{~V}$ is $15 \%$ larger than that of $\mathrm{Pt} / \mathrm{C}$. Further, the $\mathrm{Rh} / \mathrm{SiNW}$ electrode remained stable for continuous operation of 500,000 s (Supplementary Note 3).

The design concept of the $\mathrm{Rh} / \mathrm{SiNW}$ electrode was checked and verified by theoretical calculations which provided insight into the HER mechanism and verified the low $0.024 \mathrm{~V} \mathrm{dec}^{-1}$ Tafel slope obtained. The calculations support the regeneration of hydroxyl poisoning by $\mathrm{Rh}$, which contributes to the long-term stability of the $\mathrm{Rh} / \mathrm{SiNW}$ electrode. This work opens the door for a new way of producing improved electrocatalysts for HER overcoming theoretical limitations and potentially reducing the cost of hydrogen production by electrolysis. 


\section{Methods}

Materials. $\mathrm{RhCl}_{3} \cdot 3 \mathrm{H}_{2} \mathrm{O}$ was purchased from Aladdin Industrial Co.; Nafion (5 wt\%), 20 and $40 \% \mathrm{Pt} / \mathrm{C}$ catalysts from Sigma-Aldrich Co. Other reagents used were of analytical reagent grade without further purification. Doubly distilled water was used throughout the experiment.

Fabrication of metal/SiNW catalysts. Silicon nanowires obtained via oxideassisted growth method ${ }^{13}$ were etched with $5 \mathrm{ml} 5 \% \mathrm{HF}$ aqueous solution for $1 \mathrm{~min}$ to remove the $\mathrm{SiO}_{2}$ sheath and to form $\mathrm{Si}-\mathrm{H}$ bonds on their surface. The etchedSiNWs were rinsed with distilled water, and then immersed in $10 \mathrm{ml}$ of different concentrations of $\mathrm{RhCl}_{3}$ aqueous solution with stirring for $30 \mathrm{~min}$ to form $\mathrm{Rh} /$ SiNW with different contents of Rh. $29.1 \mathrm{wt} \% \mathrm{Rh} / \mathrm{SiNW}$ was immersed in excessive $5 \% \mathrm{HF}$ aqueous solution to etch the residual silicon nanowires to ultimately obtain pure Rh NPs. These NPs were used to study the electrochemical properties of pure $\mathrm{Rh} . \mathrm{Ag} / \mathrm{SiNW}$ nanocomposites was prepared by reducing silver nitrate, while ( $\mathrm{Pd}, \mathrm{Au}, \mathrm{Pt}, \mathrm{Ru}$ and $\mathrm{Re}) / \mathrm{Si}$ nanocomposites were obtained by reducing corresponding metal chloride aqueous solutions in a similar manner at the appropriate concentrations. $30.5 \mathrm{wt} \% \mathrm{Pt} / \mathrm{SiNW}$ was immersed in excessive $5 \%$ HF aqueous solution to etch the residual SiNWs to ultimately obtain pure Pt NPs.

Characterization. The structure of the samples was characterized by X-ray diffraction (Philips X'pert PRO MPD diffractometer) applying $\mathrm{Cu} \mathrm{K} \alpha$ radiation $(\lambda=0.15406 \mathrm{~nm})$. TEM and high-resolution TEM images were recorded using a FEI Tecnai F20 transmission electron microscope with accelerating voltage of $200 \mathrm{kV}$. The chemical states of the catalysts were studied by X-ray photoelectron spectroscopy performed using a Kratos AXIS UltraDLD ultrahigh vacuum surface analysis system with $\mathrm{Al} \mathrm{K \alpha}$ radiation $(1486 \mathrm{eV})$ as probe. The Brunauer-EmmettTeller (BET) specific surface area of these samples was investigated by the ASAP 2020 instrument at $77 \mathrm{~K}$. The $\mathrm{Rh}$ and $\mathrm{Si}$ contents of the catalysts were determined with Hitachi 180-80 atomic absorption spectrometer and ND-2105 trace silicate analyser. The hydrogen gas content was detected with a gas chromatograph (GC-7890T) with nitrogen as carrier gas.

Electrochemical measurements. The electrochemical measurements were carried out in a conventional three-electrode cell connected to a Princeton VersaSTAT4 electrochemistry workstation. A platinum plate with an area of $2 \mathrm{~cm}^{2}$ was used as the counter electrode, a saturated calomel electrode (SCE) was used as the reference electrode and a modified GC electrode with a diameter of $0.3 \mathrm{~cm}$, into which the studied catalysts were introduced, as the working electrode. The area of the GC electrode was calculated to be $0.0707 \mathrm{~cm}^{2}$, which was used to determine the current density in LSV. The working electrode was installed facing upward for the quick release of the evolving hydrogen gas.

For a typical fabrication of the working electrode, $2 \mathrm{mg}$ catalysts were dispersed in $1 \mathrm{ml}$ of $5: 1 \mathrm{v} / \mathrm{v}$ water-isopropanol mixed solvent with $100 \mu \mathrm{l} 0.5 \mathrm{wt} \%$ Nafion solution, and ultrasonically stirred for at least $30 \mathrm{~min}$ to reach a homogeneous suspension. Then $7.5 \mu \mathrm{l}$ of the suspension was loaded onto a GC electrode with a diameter of $3 \mathrm{~mm}$ (loading $\sim 0.193 \mathrm{mg} \mathrm{cm}^{-2}$ ). Finally, the as-prepared catalyst film was dried at room temperature.

The potentials reported in this work were versus RHE applying the calibration procedure described below. In all measurements a SCE electrode was used as the reference and the potential values were calibrated with respect to RHE. The calibration was performed in a high-purity hydrogen-saturated electrolyte with a $\mathrm{Pt}$ foil as the working electrode. In $0.5 \mathrm{M} \mathrm{H}_{2} \mathrm{SO}_{4}$ solution, $\mathrm{E}_{\mathrm{vs} \mathrm{RHE}}=\mathrm{E}_{\mathrm{vS} S \mathrm{SCE}}+0.251 \mathrm{~V}$. All the potentials reported in the manuscript were against RHE. Before any electrochemical measurement, the electrolyte solution was purified with $\mathrm{N}_{2}$ to completely remove oxygen, and stable polarization performance was recorded after 10 cycles. All data were reported without iR compensation.

Calculations. DFT calculations were carried out with the VASP code $^{18,19}$. The electron-ion interactions were described with the projector augmented wave method $^{20}$ and an energy cutoff of $400 \mathrm{eV}$. The GGA-PBE functional ${ }^{21}$ were employed to describe the exchange-correlation energy. The effect of van der Waals interactions was included with the van der Waals-DF method ${ }^{22}$. The Si(111) and $\mathrm{Rh}(111)$ surfaces were represented using periodic slabs, consisting of six $\mathrm{Si}$ and four $\mathrm{Rh}$ atomic layers, respectively. The bottom four layers of $\mathrm{Si}$ and two layers of $\mathrm{Rh}$ atoms were kept fixed. Structural relaxations were stopped when the force on each atom was smaller than $0.01 \mathrm{eV}$ per Angstrom. The transition states were determined with the climbing image nudged-elastic band method. Five structural images were inserted between the initial states and the final states in all climbing image nudged-elastic band calculations. The first Irreducible Brillouin zone was sampled using a $2 \times 2 \times 1$ Monkhorst-Pack grid for all calculations.

In the proposed mechanism, the hydrogen atoms on $\mathrm{Si}$ atoms are coming from the $\mathrm{Rh}$ surface rather than from the solution. Correspondingly, the difference of free energy $\left(\Delta \mathrm{G}_{\mathrm{H}^{*}}\right)$ is calculated by $\Delta \mathrm{G}_{\mathrm{H}^{*}}=\Delta \mathrm{E}_{\mathrm{H}}+\Delta \mathrm{E}_{\mathrm{ZPE}}-\mathrm{T} \Delta \mathrm{S}=\mathrm{G}_{\mathrm{H}-\mathrm{Si} / \mathrm{Rh}}-\mathrm{G}_{\mathrm{H}-\mathrm{Rh} / \mathrm{Si}}$. Here, $\mathrm{G}_{\mathrm{H}-\mathrm{Rh} / \mathrm{Si}}$ represents the free energy when the hydrogen atom is adsorbed on a $\mathrm{Rh}$ surface at the $\mathrm{Rh} / \mathrm{Si}$ interface (see Fig. $4 \mathrm{~b}$ ), while $\mathrm{G}_{\mathrm{H}-\mathrm{Si} / \mathrm{Rh}}$ represents the free energy when the adsorbed hydrogen is diffused on a $\mathrm{Si}$ atom at the $\mathrm{Rh} / \mathrm{Si}$ interface (see Fig. 4d). Taking into consideration that the vibrational entropy in the adsorbed state is very small ${ }^{17}$, the contribution from entropy change $(T \Delta S)$ is neglected. The
$\Delta \mathrm{E}_{\mathrm{H}}$ and $\Delta \mathrm{E}_{\mathrm{ZPE}}$ are obtained from our DFT calculations. The calculated $\Delta \mathrm{G}_{\mathrm{H}^{*}}=\Delta \mathrm{E}_{\mathrm{H}}+\Delta \mathrm{E}_{\mathrm{ZPE}}=-0.10 \mathrm{eV}$. This value is also listed in Supplementary Table 2 .

Data availability. All relevant data are available on request, which should be addressed to M.S.

\section{References}

1. Helm, M. L., Stewart, M. P., Bullock, R. M., DuBois, M. R. \& DuBois, D. L. A synthetic nickel electrocatalyst with a turnover frequency above $100,000 \mathrm{~s}^{-1}$ for $\mathrm{H}_{2}$ production. Science 333, 863-866 (2011).

2. Jaramillo, T. F. et al. Identification of active edge sites for electrochemical $\mathrm{H}_{2}$ evolution from $\mathrm{MoS}_{2}$ nanocatalysts. Science 317, 100-102 (2007).

3. Kibsgaard, J., Chen, Z. B., Reinecke, B. N. \& Jaramillo, T. F. Engineering the surface structure of $\mathrm{MoS}_{2}$ to preferentially expose active edge sites for electrocatalysis. Nat. Mater. 11, 963-969 (2012).

4. Karunadasa, H. I. et al. A molecular $\mathrm{MoS}_{2}$ edge site mimic for catalytic hydrogen generation. Science 335, 698-702 (2013).

5. Voiry, D. et al. Enhanced catalytic activity in strained chemically exfoliated $\mathrm{WS}_{2}$ nanosheets for hydrogen evolution. Nat. Mater. 12, 850-855 (2013).

6. Huang, X. et al. Solution-phase epitaxial growth of noble metal nanostructures on dispersible single-layer molybdenum disulfide nanosheets. Nat. Commun. 4, 1444 (2013).

7. Gao, M. R. et al. An efficient molybdenum disulfide/cobalt diselenide hybrid catalyst for electrochemical hydrogen generation. Nat. Commun. 6, 5982 (2015).

8. Zheng, Y. et al. Hyarogen evolution by a metal-free electrocatalyst. Nat. Commun. 5, 3783 (2014).

9. Fei, H. L. et al. Atomic cobalt on nitrogen-doped graphene for hydrogen generation. Nat. Commun. 6, 8668 (2015).

10. Hernandez-Fernandez, P. et al. Mass-selected nanoparticles of PtxY as model catalysts for oxygen electroreduction. Nat. Chem. 6, 732-738 (2014).

11. Quaino, P., Juarez, F., Santos, E. \& Schmickler, W. Volcano plots in hydrogen electrocatalysis-uses and abuses. Beilstein J. Nanotechnol. 5, 846-854 (2014).

12. Trasatti, S. Work function, electronegativity, and electrochemical behaviour of metals: III. Electrolytic hydrogen evolution in acid solutions. J. Electroanal. Chem. 39, 163-184 (1972).

13. Zhang, R. Q., Lifshitz, Y. \& Lee, S. T. Oxide-assisted growth of semiconducting nanowires. Adv. Mater. 15, 635-640 (2003).

14. Shao, M. W., Cheng, L., Zhang, X. H., Ma, D. D. D. \& Lee, S. T. Excellent photocatalysis of HF-treated silicon nanowires. J. Am. Chem. Soc. 131, 17738-17739 (2009).

15. Morales-Guio, C. G., Stern, L. A. \& Hu, X. L. Nanostructured hydrotreating catalysts for electrochemical hydrogen evolution. Chem. Soc. Rev. 43, 6555-6569 (2014).

16. Lasia, A. Electrochemical Impedance Spectroscopy and its Applications. P. 159 (Springer Science-Business Media, 2014).

17. Nørskov, J. K. et al. Trends in the exchange current for hydrogen evolution. J. Electrochem. Soc 152, J23-J26 (2005).

18. Kresse, G. \& Furthmuller, J. Efficiency of $a b$-initio total energy calculations for metals and semiconductors using a plane-wave basis set. Comput. Mater. Sci. 6 , 15-50 (1996).

19. Kresse, G. \& Furthmuller, J. Efficient iterative schemes for ab initio total-energy calculations using a plane-wave basis set. Phys. Rev. B 54, 11169-11186 (1996)

20. Kresse, G. \& Joubert, D. From ultrasoft pseudopotentials to the projector augmented-wave method. Phys. Rev. B 59, 1758-1775 (1999).

21. Perdew, J. P., Burke, K. \& Ernzerhof, M. Generalized gradient approximation made simple. Phys. Rev. Lett. 77, 3865-3868 (1996).

22. Klimeš, J., Bowler, D. R. \& Michaelides, A. Van der Waals density functionals applied to solids. Phys. Rev. B 83, 195131 (2011).

\section{Acknowledgements}

The project was supported by Major Research Plan of National Natural Science Foundation of China (No. 91433111), the National Basic Research Program of China (973 Program) (Grant No. 2012CB932903), Qing Lan Project, Collaborative Innovation Center of Suzhou Nano Science \& Technology, the Priority Academic Program Development of Jiangsu Higher Education Institutions, the Natural Science Foundation of Jiangsu Province (BK20150305).

\section{Author contributions}

L.Z. and H.L. contributed equally to the conception, planning of the project, performance of the experiments and co-wrote the manuscript. M.S. and S.-T.L. provided overall guidance in experimental design, experimental planning, data analysis and interpretation, scientific discussions throughout the project and manuscript writing. F.L. and M.S. conducted some electrochemical experiments. Y.L. performed the theoretical modelling 
and calculation. Y.L. participated in the experimental design, data analysis and the interpretation and played a major role in the manuscript writing.

\section{Additional information}

Supplementary Information accompanies this paper at http://www.nature.com/ naturecommunications

Competing financial interests: The authors declare no competing financial interests.

Reprints and permission information is available online at http://npg.nature.com/ reprintsandpermissions/
How to cite this article: Zhu, L. et al. A rhodium/silicon co-electrocatalyst design concept to surpass platinum hydrogen evolution activity at high overpotentials. Nat. Commun. 7:12272 doi: 10.1038/ncomms12272 (2016).

(c) (i) This work is licensed under a Creative Commons Attribution 4.0 International License. The images or other third party material in this article are included in the article's Creative Commons license, unless indicated otherwise in the credit line; if the material is not included under the Creative Commons license, users will need to obtain permission from the license holder to reproduce the material. To view a copy of this license, visit http://creativecommons.org/licenses/by/4.0/

(C) The Author(s) 2016 